\title{
Antibacterial activity of nano fabricated polypyrrole by cyclic voltammetry
}

\author{
S. M. Sayyah ${ }^{1}$, F. Mohamed ${ }^{1}$, and M. Shaban ${ }^{2, *}$ \\ ${ }^{1}$ Polymer Research lab, Chemistry Department, Faculty Of Science, Beni-Suef University, Beni-Suef, Egypt \\ ${ }^{2}$ Nanophotonics and Applications (NPA)Lab, Department of Physics, Faculty of Science, Beni-Suef University, \\ Beni-Suef 62514, Egypt
}

\begin{abstract}
The oxidative chemical polymerization of pyrrole was synthesized by cyclic voltammetry in acidic medium using sulfuric acid as an electrolyte at $30^{\circ} \mathrm{C}$. The synthesized polymer was characterized by SEM, and $\mathrm{XRD}$. The activity of the fabricated polymer was evaluated by agar well cut diffusion method against three bacteria: Streptococcus pneumonia (RCMB 010010), Enterococcus faecali (RCMB 010068), and Staphylococcus aureus (RCMB 010028). The polymer exhibited moderate antibacterial activity and significantly not active of antifungal.
\end{abstract}

Keywords: polypyrrole; electropolymerization; characterization; antibacterial activity

\section{Introduction}

Recently, serious infections of microbe have become a social problem. Therefore, safe extermination of microbe is very important to human health care. During the last two decades, continuous effort has been made to develop the Polymers with antimicrobial function [1]. The control of the growth of microbes such as bacteria, fungi, yeast and algae in nature is one of the fundamental concepts for the survival of higher species. Plants, animals, even microbes themselves have developed a great variety of mechanisms that keep microbes at bay [2]. In human society these control mechanisms often do not work efficiently, which makes microbial infections the number one killer in the world. The treatment of microbial infections becomes more and more difficult, because the number of resistant microbial strains as well as that of antibiotic-immune patients grows a lot faster than the number of useable antibiotics [3].

Antimicrobial polymers represent a class of biocides that has become increasingly important as an alternative to existing biocides and in some cases even to antibiotics. The working mechanism of the large number of structurally different polymers is often not fully understood. However, some of them are known for their low potential of building up resistant microbial strains [4].

Generally, polymeric antimicrobial agents have the following advantages. It is believed that they are non volatile, chemically stable, and do not permeate through the skin .As a result, the application of polymers with antibacterial activities will be a major step towards a healthier living. On the other hand, though hundreds of thousands of polymeric compounds have been prepared, few of them were of visible antimicrobial activities [5].

Polymers are being considered for a range of biomedical applications including the development of artificial muscles [6], controlled drug release [7], and the stimulation of nerve regeneration [8]. Low cytotoxicity and good biocompatibility of these materials are evident from the growth of cells on conducting polymers and from the low degree of inflammation seen in test animals over a period of several weeks [9]. Conducting polymers are redox-active because they act as reducing agents and scavenge free radicals.

Polyaminophenol was synthesized by electrochemical polymerization. Their antibacterial activities were examined and the results shown that the obtained polymer has high antibacterial activity compared with the standard. The antibacterial activity is in accordance with the literature value which shows that the polymer containing phenol derivatives with one, two, or three hydroxyl groups exhibited good antibacterial activities [10]. The high antibacterial activity could make this polymer to be as biomaterials in biological media [11] and could pay way for the development of novel drugs to control diseases and infections.

In our study, the electro-oxidation of pyrrole was carried out from aqueous $\mathrm{H}_{2} \mathrm{SO}_{4}$ medium as electrolyte using cyclic voltammetry technique. The characterization of the obtained polymer film was carried out by SEM and X-Ray diffraction. Moreover, the antibacterial activity were discussed against different species of gram positive (+) bacteria such as Streptococcus pneumoniae, Enterococcus faecalis, and Staphylococcus aureus. 


\subsection{Chemicals}

\section{Materials And Methods}

All the used chemicals were of analytical grade, supplied from Sigma-Aldrich and used as received.

\subsection{Preparation of poly pyrrole}

The poly pyrrole (PP) was synthesized by cyclic voltammetry method in acidic medium using sulfuric acid at $313 \mathrm{~K}$ and scan rate of $30 \mathrm{mvs}^{-1}$.

\subsection{Antimicrobial measurements}

The disks of Whatman filter paper were prepared with standard size $(50 \mathrm{~mm}$ diameter) and kept into 10 screw capped wide mouthed containers for sterilization. These bottles are kept into hot air oven at a temperature of $150^{\circ} \mathrm{C}$. Then, the standard sterilized filter paper disks impregnated with a solution of the test compound in DMSO $(1 \mathrm{mg} / \mathrm{mL})$ were placed on nutrient agar plate seeded with the appropriate test organism in triplicates. Standard conditions of $106 \mathrm{CFU} / \mathrm{mL}$ (Colony Forming U/mL) and $104 \mathrm{CFU} / \mathrm{mL}$ were used for antibacterial assay, respectively. Petri dishes $(9 \mathrm{~cm}$ in diameter) were used and two disks of filter paper were inoculated in each plate. The utilized test organism was Streptococcus pneumoniae (S. pneumoniae (RCMB 010010)) as example of gram positive bacteria. Ampicillin was used as a reference drug against gram positive bacteria. DMSO alone was used as a control at the same aforementioned concentration and during this, there was no visible change in bacterial growth. For bacteria, the plates were incubated at $37^{\circ} \mathrm{C}$ for $24 \mathrm{~h}$. The derivative that showed significant growth inhibition zone using the twofold serial dilution technique were further evaluated for their minimal inhibitory concentration (MIC).

\subsection{Minimal inhibitory concentration (MIC) measurement:}

The microdilution susceptibility test in Muller-Hinton Broth (Oxoid) and Sabouraud Liquid Medium (Oxoid) were used for the determination of antibacterial activity, respectively. Stock solutions of the tested compounds, were prepared in DMSO. Each stock solution was diluted with standard method broth (Difco) to prepare serial twofold dilutions of the broth containing about $106 \mathrm{CFU} / \mathrm{mL}$ of test bacteria was added to each well of 96-well microtiter plate. The sealed microplates were incubated at $37^{\circ} \mathrm{C}$ for $24 \mathrm{~h}$ for antibacterial activity in a humid chamber. At the end of the incubation period, the minimal inhibitory concentrations (MICs) values were recorded as the lowest concentrations of the substance that had no visible turbidity. Control experiments with DMSO and uninoculated media were run parallel to the test compounds under the same conditions.

\subsection{Characterization}

Scanning electron microscopic (SEM) analysis was carried out on the as-prepared polymer film deposited on Pt-working electrode surface using a JSM-T20 Electron Probe Microanalyzer (JEOL, Tokyo, Japan). The X-ray diffraction analysis (XRD), Philips 1976 Model 1390, Netherlands, was operated under the following conditions that were kept constant: $\mathrm{X}$-ray tube, $\mathrm{Cu}$; scan speed, $8 \mathrm{deg} \cdot \mathrm{min}^{-1}$; current, $30 \mathrm{~mA}$; voltage, $40 \mathrm{kV}$; preset time, $10 \mathrm{~s}$.

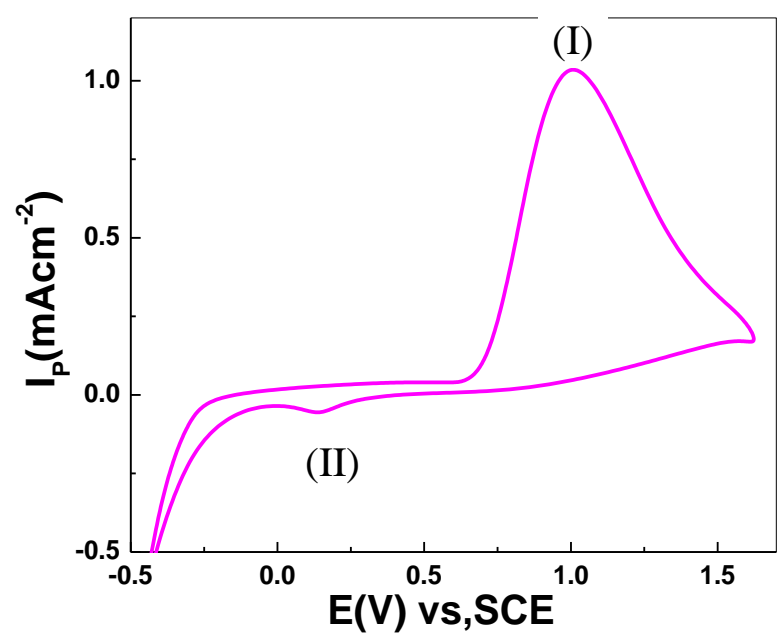

Figure.1. cyclic voltammogram of electropolymerization of $0.07 \mathrm{M}$ pyrrole on platinum electrode. 


\subsection{Electropolymerization}

\section{Results And Discussion}

Electropolymerization of pyrrole on platinum electrode from aqueous solution containing $0.6 \mathrm{M}$ $\mathrm{H}_{2} \mathrm{SO}_{4}$ and $0.07 \mathrm{M}$ of monomer at $313 \mathrm{~K}$ was studied by cyclic voltammetry at potential between -500 and $+1800 \mathrm{mV}$ (vs. SCE) with scan rate of $30 \mathrm{mVs}^{-1}$. The obtained voltammogram is represented in "Fig.1". This voltammogram exhibits an oxidation peak (I) at $1080 \mathrm{mV}$ (vs. SCE). This peak corresponds to oxidation of pyrrole to pyrrole radical-cation which then reacts with a second molecule of pyrrole leading to dipyrrole. Thus, this dimer is converted preferentially into the radical-cation which reacts with monomer leading to pyrrole trimer and finally the polymer formed. A reduction peak (II) appears at $220 \mathrm{mV}$ (vs.SCE). This peak corresponds to the reduction of the formed polymer film. [12].

\subsection{Morphology and structure of polypyrrole film}

The morphology of the synthesized nano polypyrrole (NPP) was measured by SEM and shown in "Fig.2". From this figure it was evident that the morphology of the resultant self-assembly polypyrrole is nonideal spherical aggregates. The aggregates diameter was determined to be varied from 10 to $50 \mathrm{~nm}$ and seemed to be variant in concentration. In order to check the structure of the fabricated polypyrrole, the sample was analyzed by XRD as shown in "Fig.3". This is clearly a consequence of the configuration of prepared polymer on the Pt surface as already revealed in the SEM image in "Fig.2". This XRD pattern in "Fig.3" indicates that the polymer is amorphous structure.

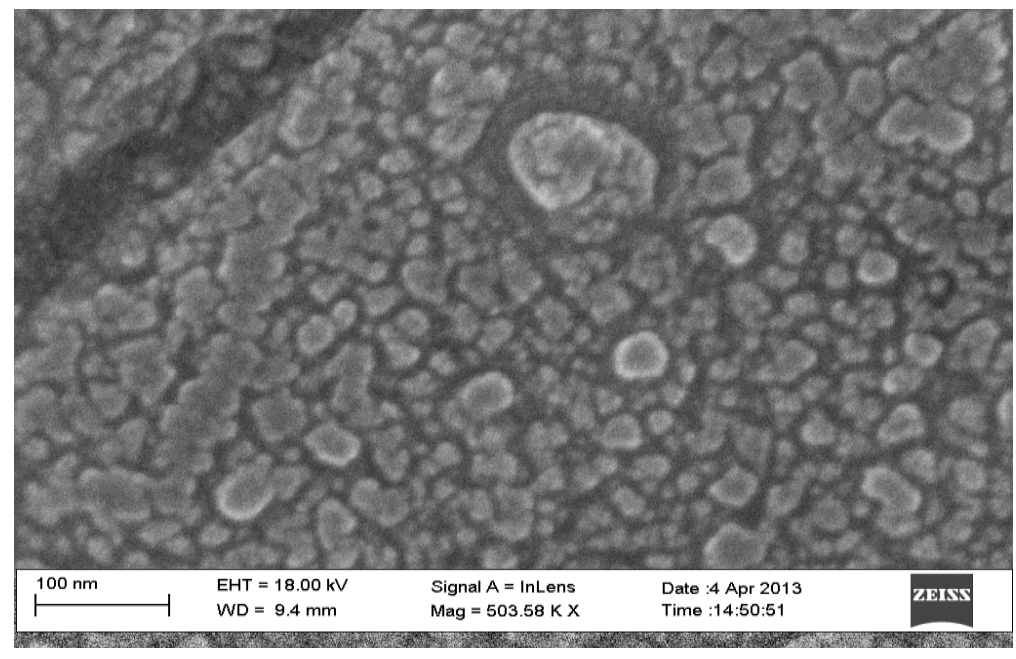

Figure. 2. SEM of polypyrrole electropolymerized on on platinum electrode.

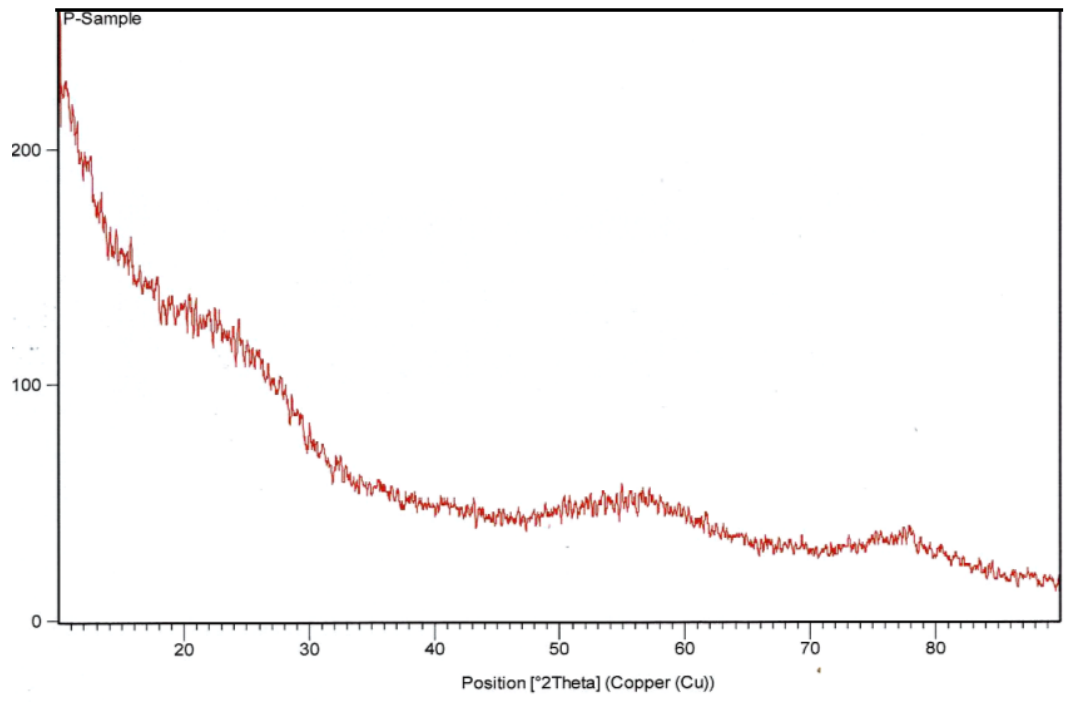

Figure. 3. XRD of polypyrrole electropolymerized on platinum electrode. 


\subsection{Antibacterial Activity}

Polypyrrole was investigated for antibacterial activity against Streptococcus pneumoniae (RCMB010010), Enterococcus faecalis (RCMB 010068) and Staphylococcus aureus (RCMB 010028) as gram positive bacteria. Agar disk diffusion method was used for the determination of the antibacterial activity Ampicillin was used as reference drug against gram positive bacteria.

Polypyrrole showed in -vitro antibacterial activity against the tested bacteria. The results of antibacterial activity of fabricated polymer using inhibition zone method are listed in "Table 1". As shown in this table, the prepared polymer showed a better antibacterial activity due to presence of NH group and aromatic ring, thus increasing its partially cationic character $[13,14]$. Consequently the positive charge was strengthened, leading to a better antibacterial activity [15]. Polypyrrole showed higher antibacterial activity against the gram positive bacteria. It causes inhibition zone diameter of $15.8 \pm 0.58,14.9 \pm 0.63$ and $15.0 \pm 1.22 \mathrm{~mm}$ for $\mathrm{S}$. pneumoniae and E. faecalis and S. aureus, respectively. This may be attributed to the cell wall of Gram positive bacteria is fully composed of peptide polyglycogen. The peptido-glycan layer is composed of networks with plenty of pores, which allow foreign molecules to come into the cell without difficulty and allows more rapid absorption of ions into the cell.

Table 1. Antimicrobial activity of poly pyrrole.

\begin{tabular}{|l|l|l|}
\hline Tested microorganisms & Polypyrrole & Standard \\
\hline Gram Positive Bacteria: & & Ampicillin \\
\hline Streptococcus pneumoniae (RCMB 010010) & $15.8 \pm 0.58$ & $23.8 \pm 0.2$ \\
\hline Enterococcus faecalis (RCMB 010068) & $14.9 \pm 0.63$ & $20.3 \pm 0.3$ \\
\hline Staphylococcus aureus (RCMB 010028) & $15.0 \pm 1.22$ & $28.3 \pm 0.1$ \\
\hline
\end{tabular}

Table 2. Antimicrobial_activity as MIC $(\mu \mathrm{g} / \mathrm{ml})$

\begin{tabular}{|c|c|c|}
\hline \multicolumn{1}{|c|}{ sample } & $\underline{\text { Polypyrrole }}$ \\
Tested microorganisms & & Ampicillin \\
\hline Gram Positive Bacteria: & 62.5 & 0.24 \\
\hline $\begin{array}{c}\text { Streptococcus pneumoniae } \\
\text { (RCMB 010010) }\end{array}$ & 125 & 3.9 \\
\hline $\begin{array}{c}\text { Enterococcus faecalis } \\
\text { (RCMB 010068) }\end{array}$ & 125 & 0.03 \\
\hline $\begin{array}{c}\text { Staphylococcus aureus } \\
\text { (RCMB 010028) }\end{array}$ & & \\
\hline
\end{tabular}

An additional evidence for the activity of polypyrrole against gram positive bacteria comes from their minimum inhibitory concentration (MIC) values. MIC is defined as the lowest concentration of an antimicrobial that will inhibit the visible growth of a microorganism after overnight incubation. The MIC value of polypyrrole against $\mathrm{S}$. pneumoniae and $\mathrm{E}$. faecalis and $\mathrm{S}$. aureus were $62.5,125$ and $125 \mathrm{Mg} / \mathrm{mL}$, respectively is represented in "Table 2". MIC values are confirmed to the noticeable antibacterial activity of the fabricated polymer where the response of the polymer to Enterococcus faecal (RCMB 010068) and Streptococcus pneumoniae (RCMB 010010) and Staphylococcus aureus (RCMB 010028).

\section{Conclusion}

Polypyrrole was synthesized electrochemically by cyclic voltammetry technique and characterized by different tools as scanning electron microscope and XRD. The self-assembly polymer is amorphous non-ideal spherical aggregates. The polymer was evaluated for its efficiency in-vitro against some species of gram positive bacteria such as Enterococcus faecal (RCMB 010068), Streptococcus pneumonia (RCMB 010010), and Staphylococcus aureus (RCMB 010028) using agar diffusion technique. The fabricated nanopolymer exhibited good antimicrobial potency with MIC values ranging from 62.5 to $125 \mathrm{Mg} / \mathrm{mL}$ as antibacterial agents. Polypyrrole displayed antbacterial activity against some tested strains almost equivalent to the standard drug, ampicillin. 


\section{References}

[1] L.U. Guiqian, W.U. Dingcai , D.Weimin1and F.U. Ruowen, Studies on the synthesis and antibacterial activities of polymeric quaternary ammonium salts from dimethylaminoethyl methacrylate , Journal of Reactive and Functional polymers, 67, $2007,355$.

[2] P. Nicolas, A .Mor, Peptides as weapons against microorganisms in the chemical defense system of vertebrates, Journal of Annual Review of Microbiology, 49, 1995, 277-304.

[3] H.M. Lode, Clinical impact of antibiotic-resistant Gram-positive pathogens, Journal of Clinical Microbiology and Infection, 15, 2009, 212-217

[4] N.M. Milovic, J.Wang,K.Lewis, A.M. Klibanovm, Immobilized $N$-alkylated polyethylenimine avidly kills bacteria by rupturing cell membranes with no resistance developed,Journal of Biotechnoogyl and Bioengineering, 90, 2005, 715-722.

[5] B. Dizman, M.O. Elasri, L.J. Mathias, ScienceSynthesis and antimicrobial activities of new water-soluble bis-quaternary ammonium methacrylate polymers, Journal of Applied Polymer, 94, 2004, 635.

[6] G. Han, G.Shi, Conducting polymer electrochemical actuator made of high-strength three-layered composite films of polythiophene and polypyrrole, Journal of Sensors and Actuators B, , 99,2004, 525.

[7] J.M. Pernaut, J.R. Reynolds, use of conducting electroactive polymers for drud delivery and sensing of bioactive molecules .A redox chemistry approach ,Journal of Physical Chemistry B, 104, 2000, 4080.

[8] L.A.P.Kane-Maguire, G.G. Wallace, Communicating with the building blocks of life using organic electronic conductors, Journal of Synthetic Metals, 119, 2001, 39.

[9] S. Kamalesh, P. Tan, J. Wang, T. Lee, E.T. Kang, C.H. Wang, Biocompatibility of electroactive polymers in tissues, Journal of Biomedical Materials Research, 52, 2000, 467.

[10] T. Nonaka, Y. Uemura, K. Ohse, S. Kurihara, Preparation of resins containing phenol derivatives from chloromethylstyrenetetraethyleneglycol dimethacrylate copolymer beads and antibacterial activity of resins, Journal of Applied Polymer Science, 66, 1997,1621 .

[11] G.Thenmozhi,D. J. Kumar, M. Gopalswamyb and J. R. Santhi. . Synthesis, characterisation and biological applications of conducting poly (p- amino phenol) and its nano compound ,Journal of Der Pharma Chemica, 3 (4), 2011, 116-126

[12] G. Arslan, B. Yazici, Me. Erbil, The effect of $\mathrm{pH}$, temperature and concentration on. electrooxidation of phenol, Journal of Hazardous Materials B124, 2005,37-43.

[13] M.Kong, X. G. Chen, K. Xing, H. J. Park, Antimicrobial properties of chitosan and mode of action: A state of the art review, Journal of Food Microbiology, 144, 2010, 51-63

[14] T. Nonaka, Y. Uemura, K. Ohse, S. Kurihara, Preparation of resins containing phenol derivatives from chloromethylstyrenetetraethyleneglycol dimethacrylate copolymer beads and antibacterial activity of resins, Journal of Applied Polymer Science, 66, 1997, 1621.

[15] N. A. Mohamed, M. W. Sabaa, A. H. El-Ghandourb, M. M. Abdel-Azizc and O.F. Abdel-Gawad , Quaternized N-substituted carboxymethyl chitosan derivatives as antimicrobial agents, .International Journal of Biological Macromolecules, 60, 2013, 156164. 